\title{
Comparative Analysis of Vehicle Make and Model Recognition Techniques
}

\author{
Faiza Ayub Syed \\ Department of Software Engineering, University of Engineering \& Technology, Taxila, Pakistan \\ Email: faizaayubsyed@yahoo.com \\ Malik Usman Dilawar \\ Department of Computer Engineering, Center for Advance Studies in Engineering, Islamabad, Pakistan \\ Email: ud_malik@hotmail.com \\ Ali Javed \\ Department of Software Engineering, University of Engineering \& Technology, Taxila, Pakistan \\ Email: ali.javed@uettaxila.edu.pk
}

\begin{abstract}
Vehicle Make and Model Recognition (VMMR) has emerged as a significant element of vision based systems because of its application in access control systems, traffic control and monitoring systems, security systems and surveillance systems, etc. So far a number of techniques have been developed for vehicle recognition. Each technique follows different methodology and classification approaches. The evaluation results highlight the recognition technique with highest accuracy level. In this paper we have pointed out the working of various vehicle make and model recognition techniques and compare these techniques on the basis of methodology, principles, classification approach, classifier and level of recognition. After comparing these factors we concluded that Locally Normalized Harris Corner Strengths (LHNS) performs best as compared to other techniques. LHNS uses Bayes and K-NN classification approaches for vehicle classification. It extracts information from frontal view of vehicles for vehicle make and model recognition.
\end{abstract}

Index Terms - Make and Model Recognition (MMR), Vehicle Make and Model Recognition (VMMR).

\section{INTRODUCTION}

Many vision based automatic vehicle detection and classification techniques are in common practice nowadays. A widespread usage of these techniques seems to appear in different systems like access control systems, traffic control and monitoring systems, security systems and surveillance applications. The significance of vehicle based authentication technologies is rapidly increasing with rising trend in security awareness. A hot topic in the domain of intelligent transport system is VMMR [1]. Different object classification techniques are employed to recognize vehicle make and model [2]. Major research has been carried out in area of vehicle classification [3]. The vehicle classification algorithms distinguish vehicles on the basis of its category [4]. Major types of vehicle categories include: 1. Heavy weight, 2. Intermediate, 3. Light weight. However, classification algorithms identify the make and model of vehicles that lies within a given category. At present several vehicle recognition systems are being used in various applications.

A relative new area of research in vehicle based authentication is Vehicle MMR. The initiative behind vehicle MMR is to extract the required features of an image for recognizing the make and model of vehicle [3]. For vehicle type recognition generally two approaches are used: Model based and Appearance based. The standard model based approach to object recognition starts from 3D models of the object [5]. Recognition is done through image matching. However, the downside of model based approach is their dependency on feature extraction which sometimes gives error prone results. On the other hand the appearance based model focus on photometric properties rather than feature extraction. The recognition process defined for vehicle type consists of image extraction, feature extraction and image classification shown in fig 1 [5].

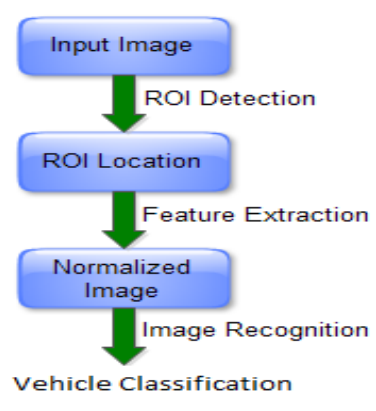

Figure 1. Recognition Process

Some of vehicle recognition techniques for identifying vehicle make and model are mentioned in literature. Common approaches to vehicle MMR is feature location and matching using Scale Invariant Transform (SIFT) [6] localized feature detection through Contourlet transform [3], Harris Corner Strength for recognizing make and 
model using k-NN and Naive Bayes Classifier [7], feature detection and description on the basis of illumination using Local Energy Shaped Histogram (LESH) [8], recognizing features by calculating feature gradients using match refinement [9], Speeded-up Robust Feature Detector (SURF) and Support Vector Machine (SVM) algorithm [10]. The computational cost, speed and accuracy achieved through these approaches vary from one another.

The rest of the paper is organized as follows. Section II contains literature review which covers different VMMR techniques. Commonly used recognition techniques are discussed in section III. Section IV contains the evaluation and comparison of discussed techniques. For this purpose a comparison table is maintained for easy understanding. Section V is the conclusion.

\section{LITERATURE REVIEW}

Psyllos and Kayafas [6] proposed an enhanced SIFT based feature matching scheme for detecting vehicle logo images. SIFT detects the image features with invariant transforms i.e. the features are invariant to scale, rotation and illumination. The recognition method entails a license plate location module followed by edge detection and symmetry based matching for logo identification. For enhancing the recognition process, they employ a multiple match method by grouping images where similar scenes are portrayed instead of taking a single image or single feature match (SFM). All SIFT based features are detected through this process. This multiple feature matching method is also known as merged feature matching (MFM). Verification of images is done through generalized Hough transform. They achieve an overall success rate of 91 percent for this process.

Greg and nick [7] explored a new approach namely Harris corner strengths for recognizing vehicle make and model. They inspect various cars images by recursively partitioning them into quadrants and calculating the feature strengths in these quadrants and then carrying out the summation and local normalization of quadrants feature strengths in a recursive hierarchical way. They probe in two kinds of classification approaches; knearest-neighbor and Naive-Bayes classification approaches. They tested 10 different classification systems by using a combination of various feature extraction methods and classification techniques. The feature extraction methods include: Canny edge detection, Square mapped gradients (SMG), Improved Square mapped gradients (ISMG), Harris corner strengths and locally normalized Harris strengths (LNHS). The classification methods include: 1-nearest-neighbor, 3nearest-neighbor and Naive Bayes classifier. The results of classification proves that LNHS in combination with $\mathrm{k}-\mathrm{NN}$ classifier perform better as compared to SMG and others because in LNHS due to smaller feature vector size the compactness and speed of detection is faster. While carrying out experiments on the vehicle dataset they achieve an accuracy level of 96 percent with this system.
Sarfraz and Saeed [8] presented a probabilistic Bayesian framework for vehicle make and model recognition. The framework requires an incoming test image of vehicle to be compared with an exactly matched image in database. The match is found on the basis of appearance, illumination and scale etc. For better consideration of these factors they propose a feature description Local Energy based Shape Histogram (LESH) where the features are represented in similarity feature matrix. With the help of this technique the image shape is encoded in presence of high illumination and noise by detecting the interest points in images with high reliability where encoding of images refers to feature extraction. LESH provides a posterior match method for feature matching. The posterior of test image is computed by calculating its similarity with each of the vehicle image feature vectors present in database. They employ a Gaussian model for approximating the similarities and dissimilarities between test image and images registered in database. The vehicle type in database for which the posterior is maximum, is considered as a best match found for the test image. Using this method they achieve an accuracy level above 94 percent.

Petrovic and Cootes [9] represented a system for automatic vehicle make and model recognition from frontal images of cars through match refinement in two stages. The system works by extracting feature gradients from indicated points in images. During the first stage a list of potential candidate classes is made by using the gradient feature vectors. The results of first stage are filtered through match refinement algorithm. The algorithm finds an exact match by optimizing object pose and normalization of feature vectors by refining the vehicle structure samples. With this system they achieve a high verification results and identification rate of 94.4 percent. This system aims to provide security and surveillance applications.

Baran, Glowacz and Matiolanski [10] presents a real time approach; for vehicle make and model recognition. In the approach they use two methods for recognizing images. First method uses a combination of speeded up robust feature (SURF) detector and support vector machine (SVM) algorithm. Second method employs a visual content classification (VCC) algorithm. This classification method speeds up the recognition process as well as provides greater accuracy. Both methods consist of training and testing phases. During testing phase calculations are performed over some reference database images. The feature vectors are calculated and stored in binary format for achieving better results with lower computational costs. During testing phase the query image is classified and its descriptors are calculated using SURF. Using both methods they achieve a greater level of accuracy.

AbdelMaseeh and Badreldin [11] suggested an efficient method for vehicle MMR by combing the local and global information of images. This approach addresses the problems that arise due to category mismatch, illumination and pose variation in vehicles. The algorithm works by creating a sketch of image 
present in reference database. The sketch is in the form of an edge map. A global descriptor is computed for each selected edge. For local edge points of an image local descriptors are calculated. The appearance and local shape descriptors calculated are then matched with local descriptors in the reference database. The experimental results prove that this approach improves the classification rate accuracy to a much higher level.

Psyllos and Kayafas [12] presented a novel approach for vehicle MMR with a rapid color recognition scheme for efficient results. The approach uses license plate recognition scheme along with masking and segmentation of vehicle frontal images for recognizing vehicle make. Simple histogram technique is used for recognizing vehicle color. For classification purpose they use a probabilistic neural network for logo detection and pattern matching. Using this classification scheme a recognition rate of 85 percent is achieved. The color recognition method speeds up the process because of its faster processing time with 90 percent accuracy. However, the research approach is limited to small range of vehicle models.

Zafar, Edirisinghe and Acar [3] proposed a contourlet based vehicle MMR approach. The approach decomposes the images using contourlet transform in two successive stages using Laplacian filter scheme. The contourlet features are extracted through the feature map. Finally SVM is used for classification purpose. The experimental results and evidences show that the total accuracy level using this approach is about 94 percent. Also this approach leads to reduction in computational cost and speeds up the classification process. They conclude that SVM classifier performs well as compared to k-NN classifier.

\section{CURRENT VEHICLE RECOGNITION TECHNIQUES}

Vehicle MMR has variety of applications in different fields such as traffic monitoring, security, and surveillance outside parking lots and buildings. Vehicle MMR proves to be best for checking against fraudulent and intrusive use of vehicles. Various techniques are available for vehicle MMR. Each technique has its own criteria for recognizing vehicles. Current recognition techniques use different algorithms for vehicle classification, detection and recognition. In this research work we have discussed 8 vehicle recognition techniques. These techniques help in detecting vehicles more efficiently and effectively. The techniques are as follows:

\section{A. VMMR through Frontal Images of Cars}

This technique makes use of frontal images of cars for detecting vehicles [7]. It introduces a novel approach based on Harris Corner Strengths. The technique works by recursively dividing images into quadrants i-e two rows and two columns and manually marking the region of interest. The feature extraction methods include: Canny edge detection, Square mapped gradients (SMG), Improved Square mapped gradients (ISMG), Harris corner strengths and locally normalized Harris strengths (LNHS). The classification methods include: 1-nearestneighbor, 3-nearest-neighbor and Naive Bayes classifier. The different feature detection techniques helps in better evaluation of results.

Major steps for this system are shown in fig 2. The system first takes an input image with pose variation. For ROI selection points are manually marked on the number plate in parallelogram shape. The image is normalized using eight depth of field transformation. Feature extraction is done through different methods including 1pixel thickened canny edge detection, Improved Square mapped gradients (ISMG) and Harris corners with k-NN and Naive Bayes being classifiers. The results of evaluation shows that using canny edge with 1-NN the classification rate is about 79.1 percent and with $3-\mathrm{NN}$ it is about 81.4 percent. For Improved Square mapped gradients (ISMG) with $1-\mathrm{NN}$ the classification rate is about 91.0 percent and with $3-\mathrm{NN}$ it is about 89.8 percent. For Harris corners the classification rate is nearly equal to that of canny edge approach. By applying the scheme of recursive division the Harris corners are localized to small patches. Using Localized Harris Corner Strengths with 1-NN classifier the classification rate is about 94.9 percent with Naive Bayes classifier this rate raises to an accuracy level of 96.0 percent. The results of using these techniques deduce that LHNS outperforms all other extraction methods while k-NN and Bayes classifier has almost same results with marginal change.

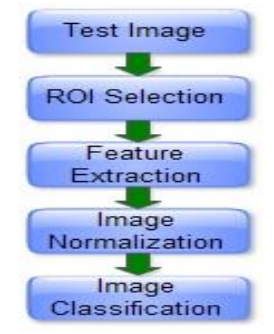

Figure 2. VMMR through Frontal Images of Cars

\section{B. Bayesian Prior Models for VMMR}

This technique makes use of an efficient probabilistic approach to vehicle MMR [8]. The approach is invariant to illumination. The steps for this recognition scheme are shown in fig 3.

The system requires an incoming test image of vehicle to be compared with an exactly matched image in database. The match is found on the basis of appearance, illumination and scale etc. When the exact match is found, ROI is selected and the image is normalized according to scale. The next stage is of feature extraction. For this purpose they propose a feature description Local Energy based Shape Histogram (LESH) where the features are represented in similarity feature matrix. With the help of this technique the image shape is encoded in presence of high illumination and noise by detecting the interest points in images with high reliability where encoding of images refers to feature extraction. LESH provides a 
posterior of test image is computed by calculating its similarity with each of the vehicle image feature vectors present in database. The vehicle type in database for which the posterior is maximum, is considered as a best match found for the test image. Using this method they achieve an accuracy level above 94 percent.

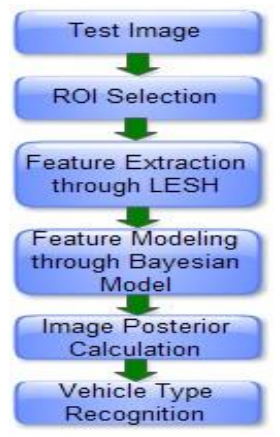

Figure 3. Bayesian Prior Models for VMMR

\section{SIFT-based Enhanced Matching Scheme for VMMR}

This research is based on enhanced SIFT based feature matching scheme for recognizing vehicle make [6]. The system first takes an input image than the image is converted to grey scale. The next step is creation of vehicle mask. The logo portion is selected for recognizing the vehicle make. The exact position of logo in vehicle mask is determined using a method based on phase congruency. Phase congruency is implemented for segmentation and detection of exact logo location whereas for feature detection SIFT detector is used. For enhancing the recognition process, they employ a multiple match method by grouping images where similar scenes are portrayed instead of taking a single image or single feature match (SFM). All SIFT based features are detected through this process. This multiple feature matching method is also known as merged feature matching (MFM). Verification of images is done through generalized Hough transform. They achieve an overall success rate of 91 percent for this process.

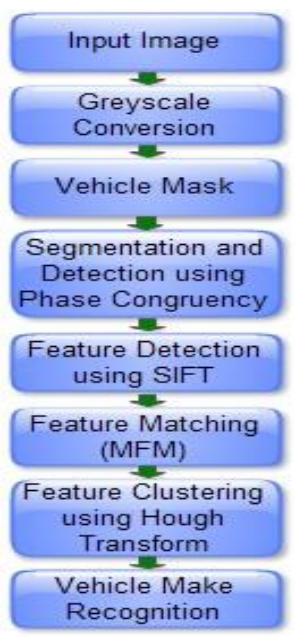

Figure 4. SIFT-based Enhanced Matching Scheme for VMMR

\section{VMMR through Match Refinement}

This research is based on vehicle recognition through match refinement [9]. The match refinement algorithm highly discriminate the features with optimum pose. The working of system is shown in fig 4 . The system first takes an input image. Vehicle frontal structure is covered in ROI i.e. license plate portion. Group of corner features are defined to be allocated for license plate. The next phase is of feature extraction. Cropping and downsampling of ROI is done for extracting features. The structure is normalized and classified using match refinement algorithm. Both match refinement and pose optimization tends to improve the performance level of recognition. Using this scheme an identification rate of 94.4 percent is achieved.

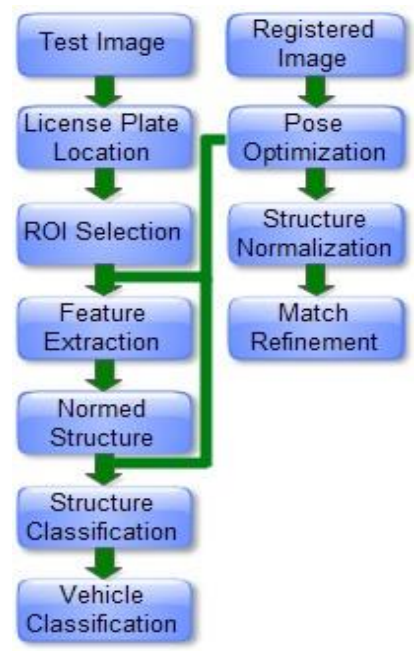

Figure 5. VMMR through Match Refinement

\section{E. Real time Vehicle MMR}

In this research a real time approach for vehicle MMR is defined [10]. This approach makes use of SURF detector. The structure of the system is shown in fig 5 . The system takes in two separate datasets for where one is the training set containing images from a reference database and the other set is used as a testing set consisting of query image. Both datasets contains images that are collected under varying light conditions. In training set, for each reference database image SURF descriptor is computed. Then all these descriptors are collected and clustered using k-means methodology. The set of clusters then takes the form of vocabulary. The number of feature vector occurrence is calculated using Sparse Vector of Occurrence Count Method (SVoOC). At the end of training phase, SVM model is created using SVM algorithm, this divides the input images into fixed number of classes. The first step in testing phase is of reading the related XML files from SVM model. The next step loads the query image. SURF descriptors are calculated for the query image and these images are then assigned to clusters in vocabulary. This results in new SVoOC calculation. Then the query image class is calculated using SVM model. 


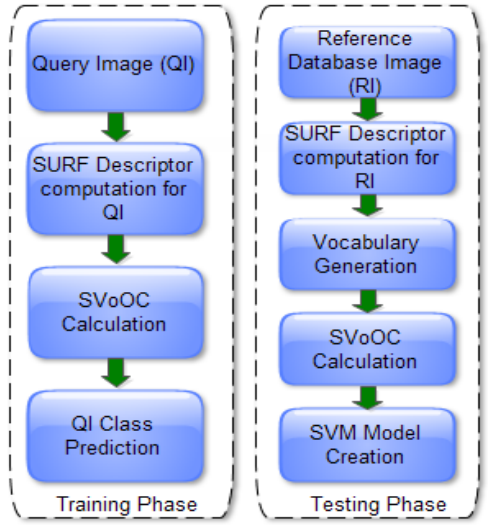

Figure 6. Real time Vehicle MMR

\section{F. Vehicle MMR through Localized Contourlet Transform}

This research is based on localized feature detection method [4]. The structure of the system is shown in fig 6 . The system first takes images from training and test sets. In preprocessing phase the frontal view of images are cropped and normalized to a size of $128 \times 128$ pixels. The next step is decomposition of images using contourlet transform. Contourlet transformation is done in two stages using Laplacian filter scheme. The Laplacian pyramid divides the image into $\mathrm{L}+1$ equal scale rank. Using directional filter cache each scale rank is then decomposed in to $\mathrm{D}$ directional bands, thus, resulting in a total of LxD bands. Feature extraction is done by localizing and extracting features from band pass images directional feature map. Finally, a Support Vector kernel is used for mapping the data from the input to the output feature space.

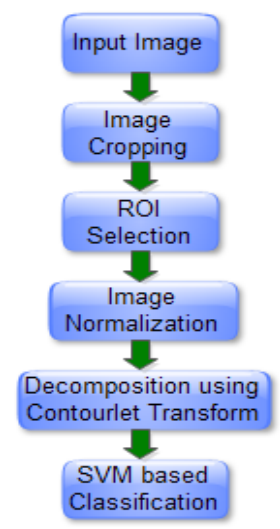

Figure 7. Vehicle MMR through Localized Contourlet Transform

\section{G. Vehicle MMR using Color Recognition Scheme}

This research is related to vehicle MMR using color recognition scheme [12]. This technique uses license plate recognition scheme along with masking and segmentation of vehicle frontal images for recognizing vehicle make. Simple histogram technique is used for recognizing vehicle color. The working of system is shown in fig 7 . The system first takes a colored image as input and performs conversion to grey scale followed by license plate recognition and segmentation using $\mathrm{W}$-mask and H-Mask where $\mathrm{H}$ and $\mathrm{W}$ denotes the height and width of the license plate. Phase congruency methodology is applied for vehicle mask segmentation. From the segmented image the red, green and blue components are selected and histogram is created for each component. The color recognition scheme makes use of these histograms along with Euclidean matrix for better results. A probabilistic neural network for vehicle manufacture recognition and pattern matching is used for classification purpose. A SIFT detector is used for calculating feature descriptors of an image. These SIFT measurements are than used for recognizing vehicle model. Using this classification scheme a recognition rate of 85 percent is achieved. The color recognition method speeds up the process because of its faster processing time with 90 percent accuracy.

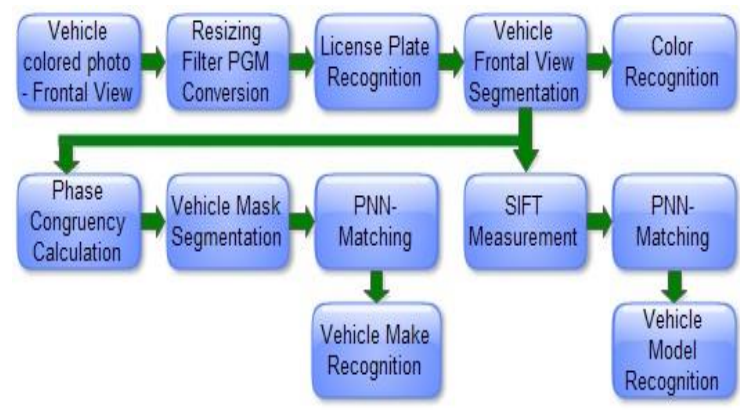

Figure 8. Vehicle MMR using Color Recognition Scheme

\section{H. Vehicle MMR by combining Local and Global Cues}

This research is based on vehicle MMR by combing the local and global information of images contained inside a category [11]. This approach addresses the problems that arise due to category mismatch, illumination and pose variation in vehicles. The structure of the system is shown in fig 8 . The system works by taking input image as query image and gallery image in which human expert labels the discriminative information on images. For each of the query image the features are extracted by sketching an edge map for visually discriminative parts. A global descriptor is computed for each selected edge. For local edge points of an image, local descriptors are calculated. Since local descriptors cannot capture all distinctive features so a combination of local shape descriptors and appearance descriptors are used for extracting required information from images. The correspondence between images is found using a weighted sum of local and global descriptors. The match between query image and gallery image is found using a voting method based on KDD-trees. Compared to manual segmentation this segmentation approach improves the classification rate accuracy to a much higher level. 


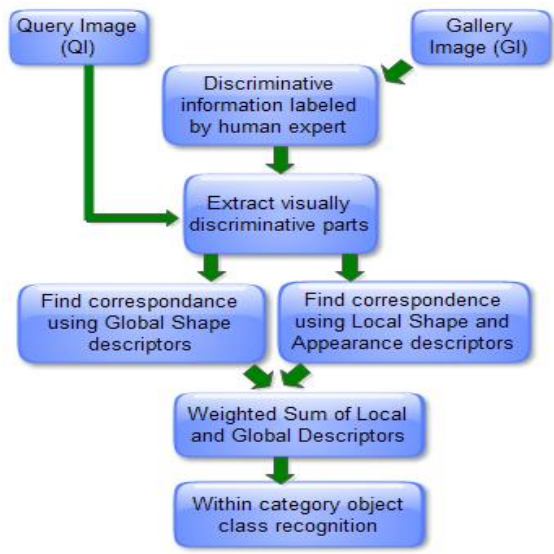

Figure 9. Vehicle MMR by combining Local and Global Cues

\section{COMPARATIVE ANALYSIS OF VMMR TECHNIQUES}

In this section we analyze the VMMR techniques discussed in previous section. Table 1 indicates the relative comparison of VMMR techniques on the basis of different parameters. From the analysis and comparison of these techniques we conclude that Locally Normalized Harris Corner Strengths (LNHS) approach is better than other approaches applied for VMMR as LNHS performs faster and have a recognition accuracy level of 96 percent. Figure 2 above shows important steps of LHNS. These steps are described below.

\section{A. ROI Selection and Normalization}

The first step is to take an input image of vehicle i.e. frontal view of a car. The next step is to define the region of interest. For this purpose the license plate corners are marked in such a way that they form a parallelogram. Corners are mapped using a homographic technique. For stable mapping Depth of Field (DOF) should be 6 or 8 . Mapping technique helps in normalizing the scale and position.

\section{B. Feature Extraction}

The feature extraction methods applied for extracting feature vectors. The methods used for feature extraction includes Canny edge detection, Square Mapped Gradients, Harris Corner Strengths, and Locally Normalized Harris Corner Strength (LHNS). LHNS technique works by recursively dividing images into quadrants i.e. two rows and two columns.

\section{Classification}

The classification approaches used are k-NN and Naive Bayes Classifier. The first classifier k-NN is an effective method of classification. The success of classification depends on the value of $k$ which is automatically determined [13]. The second classification approach used is Naive Bayes classifier. This classifier works by implementing Bayes theorem in order to evaluate the probability of occurrence of feature vectors. This classifier speeds up the recognition process.

Each recognition technique has its own methodology and principles. The recognition rate or accuracy levels of all techniques are different based on methods and principles deployed. Each technique uses a set of vehicles on which experiments are performed to get the accuracy level. The complete detail of 8 recognition techniques is outlined in Table I.

\section{TABLE I. COMPARATIVE ANALYSIS OF VMMR TECHNIQUES}

\begin{tabular}{|c|c|c|c|c|c|}
\hline $\begin{array}{l}\text { Recognition } \\
\text { Technique }\end{array}$ & $\begin{array}{l}\text { Methods \& } \\
\text { Principles }\end{array}$ & $\begin{array}{c}\text { Classification } \\
\text { Approaches }\end{array}$ & Classifiers & $\begin{array}{l}\text { Total Vehicle } \\
\text { Samples }\end{array}$ & $\begin{array}{l}\text { Accuracy } \\
\text { Level }\end{array}$ \\
\hline \multirow{4}{*}{$\begin{array}{l}\text { VMMR through } \\
\text { frontal images of } \\
\text { cars }\end{array}$} & Canny edge & \multirow{4}{*}{$\begin{array}{l}\text { K-NN, } \\
\text { Naive Bayes }\end{array}$} & \multirow{4}{*}{$\begin{array}{l}\text { Peugeot207, } \\
\text { VWGolfMk3, } \\
\text { Mini Cooper, } \\
\text { HondaJazzMk1, } \\
\text { FordFiestaMk4 }\end{array}$} & \multirow{4}{*}{$\begin{array}{l}262 \text { frontal } \\
\text { images of cars }\end{array}$} & \multirow{4}{*}{$\begin{array}{l}96.0 \% \text { using } \\
\text { LHNS and } \\
\text { Bayes classifier } \\
94.9 \% \text { using } \\
\text { LHNS and K- } \\
\text { NN classifier }\end{array}$} \\
\hline & $\begin{array}{l}\text { Square mapped } \\
\text { Gradients }\end{array}$ & & & & \\
\hline & $\begin{array}{l}\text { Harris Corner } \\
\text { Strengths }\end{array}$ & & & & \\
\hline & $\begin{array}{l}\text { Locally } \\
\text { normalized } \\
\text { Harris corner } \\
\text { strengths }\end{array}$ & & & & \\
\hline $\begin{array}{l}\text { Bayesian Prior } \\
\text { Models for } \\
\text { VMMR }\end{array}$ & LESH & $\begin{array}{l}\text { Bayesian } \\
\text { model }\end{array}$ & $\begin{array}{l}\text { Honda civic, } \\
\text { Toyota Corolla, } \\
\text { Audi, Fiat }\end{array}$ & $\begin{array}{l}300 \text { frontal view } \\
\text { images of } 25 \\
\text { different vehicle } \\
\text { types. Training } \\
\text { set contains } 100 \\
\text { images and } 25 \\
\text { test set images }\end{array}$ & $\begin{array}{lr}\text { Above } & 94 \% \\
\text { using } & \text { Bayes } \\
\text { Model } & \end{array}$ \\
\hline
\end{tabular}




\begin{tabular}{|c|c|c|c|c|c|}
\hline $\begin{array}{c}\text { SIFT-based } \\
\text { Enhanced } \\
\text { Matching Scheme } \\
\text { for VMMR }\end{array}$ & $\begin{array}{lr}\text { SIFT } & \text { based } \\
\text { feature } & \text { matching } \\
\text { scheme, multiple } \\
\text { image } \\
(\mathrm{MFM}), \quad \text { match } \\
\text { image map(SFM) }\end{array}$ & $\begin{array}{l}\text { NN classifier, } \\
\text { PNN classifier }\end{array}$ & $\begin{array}{lr}\text { Alfa } & \text { Romeo, } \\
\text { Audi, } & \text { BMW, } \\
\text { Citroen, } & \text { Fiat, } \\
\text { Peugeot, } & \text { Renault, } \\
\text { Seat, Toyota, VW }\end{array}$ & $\begin{array}{l}1200 \text { frontal } \\
\text { images }\end{array}$ & $\begin{array}{l}\text { SFM: } 91 \% \\
\text { MFM: } 94 \%\end{array}$ \\
\hline $\begin{array}{l}\text { Recognition } \\
\text { Technique }\end{array}$ & $\begin{array}{l}\text { Methods \& } \\
\text { Principles }\end{array}$ & $\begin{array}{l}\text { Classification } \\
\text { Approaches }\end{array}$ & Classifiers & $\begin{array}{l}\text { Total Vehicle } \\
\text { Samples }\end{array}$ & $\begin{array}{l}\text { Accuracy } \\
\text { Level }\end{array}$ \\
\hline $\begin{array}{l}\text { VMMR through } \\
\text { Match Refinement }\end{array}$ & $\begin{array}{l}\text { Recognition with } \\
\text { gradient } \\
\text { structures }\end{array}$ & MLP network & $\begin{array}{l}77 \text { distinct classes } \\
\text { of cars including } \\
\text { Ford Puma, } \\
\text { Peugeot } 406 \text { etc }\end{array}$ & $\begin{array}{l}1000 \quad \text { frontal } \\
\text { images } \\
\text { containing } 77 \\
\text { different vehicle } \\
\text { types }\end{array}$ & $\begin{array}{l}94.4 \% \text { using } \\
\text { MLP network }\end{array}$ \\
\hline Real time VMMR & $\begin{array}{l}\text { Recognition } \\
\text { using SURF } \\
\text { detector }\end{array}$ & $\begin{array}{l}\text { SVM } \\
\text { classifier }\end{array}$ & $\begin{array}{l}\text { Distinct classes of } \\
\text { cars }\end{array}$ & $\begin{array}{l}1360 \text { images } \\
\text { with } 17 \text { different } \\
\text { car models in } \\
\text { training dataset. } \\
2499 \text { images in } \\
\text { test dataset. }\end{array}$ & $\begin{array}{l}91.7 \% \quad \text { SVM } \\
\text { classifier }\end{array}$ \\
\hline $\begin{array}{l}\text { VMMR through } \\
\text { Localized } \\
\text { Contourlet } \\
\text { Transform }\end{array}$ & $\begin{array}{l}\text { Contourlet } \\
\text { transformation }\end{array}$ & $\begin{array}{l}\text { SVM } \\
\text { classifier }\end{array}$ & $\begin{array}{l}300 \text { frontal views } \\
\text { of cars }\end{array}$ & $\begin{array}{l}\text { Database of } 300 \\
\text { images }\end{array}$ & $\begin{array}{l}96 \% \quad \text { using } \\
\text { SVM classifier }\end{array}$ \\
\hline $\begin{array}{l}\text { VMMR using } \\
\text { Color Recognition } \\
\text { Scheme }\end{array}$ & $\begin{array}{l}\text { Color recognition } \\
\text { scheme, mask } \\
\text { segmentation, } \\
\text { phase congruency }\end{array}$ & PNN classifier & $\begin{array}{l}11 \text { distinct classes } \\
\text { of cars }\end{array}$ & $\begin{array}{l}110 \quad \text { vehicle } \\
\text { images }\end{array}$ & $\begin{array}{l}90 \% \text { accuracy } \\
\text { using color } \\
\text { recognition } \\
\text { scheme }\end{array}$ \\
\hline $\begin{array}{c}\text { VMMR by } \\
\text { combining Local } \\
\text { and Global Cues }\end{array}$ & $\begin{array}{l}\text { Recognition } \\
\text { within category } \\
\text { using global and } \\
\text { local information }\end{array}$ & $\begin{array}{l}\text { K-NN } \\
\text { classifier }\end{array}$ & $\begin{array}{l}10 \text { different } \\
\text { models of sedan }\end{array}$ & $\begin{array}{lr}10 \text { cars each } \\
\text { having } 3 \\
\text { different images }\end{array}$ & $\begin{array}{l}79.09 \%-\text { no } \\
\text { manual } \\
\text { segmentation }\end{array}$ \\
\hline
\end{tabular}

\section{CONCLUSION}

Compared to appearance based model recognition approaches like VMMR by combining Local and Global Cues, and VMMR using Match refinement the model based recognition approaches perform better. This research paper is related to comparative analysis of 8 vehicle recognition techniques. On the basis of parameters like methodology, principles, classification approaches, classifiers and recognition rate; we conclude that the model based recognition approach i.e. Locally Normalized Harris Corner Strengths (LHNS) is an efficient approach for recognizing vehicles. For recognition vehicle frontal images are taken. These images are collected under various weather conditions. It uses Bayes and K-NN classifiers for vehicle classification. The accuracy level using this approach is $96.0 \%$ which is better as compared to other techniques.

\section{REFERENCES}

[1] Hongchao Zhang, Xuezhong Xiao, Qian Zhao, "Vehicle Make and Model Recognition with Unfixed Views", in proceedings of Chinese Conference on Pattern Recognition (CCPR), IEEE, 2010.

[2] David Anthony Torres, "More Local Structure Information for Make-Model Recognition", 2005.

[3] I.Zafar, E.A.Edirisinghe, B.S.Acar, "Localised Contourlet Features in Vehicle Make and Model Recognition", SPIE-IS\&T, 2009.

[4] Kanwal Yousaf, Arta Iftikhar, Ali Javed, "Comparative Analysis of Automatic Vehicle Classification Techniques: A Survey", IJIGSP, 2012.

[5] Michal Conos, "Recognition of vehicle make from a frontal view", 2007. 
[6] Apostolos P. Psyllos, Christos-Nikolaos E. Anagnostopoulos, Eleftherios Kayafas, "Vehicle Logo Recognition Using a SIFT-Based Enhanced Matching Scheme", IEEE, June 2010.

[7] Greg Pearce, Nick Pears, "Automatic Make and Model Recognition from Frontal Images of Cars", IEEE, 2011.

[8] M.Saquib Sarfraz, Ahmed Saeed, M.Haris Khan, Zahid Riaz, "Bayesian Prior Models for Vehicle Make and Model Recognition", ACM, 2009.

[9] V.S.Petrovic, T.F.Cootes "Vehicle Type Recognition with Match Refinement", " in proceedings of Seventeenth International Conference on Pattern Recognition (ICPR), IEEE, 2004.

[10] Remigiusz Baran, Andrzej Glowacz, Andrzej Matiolanski, "The efficient real-and non-real-time make and model recognition of cars", Springerlink, June 2013.

[11] Meena AbdelMaseeh, Islam Badreldin, Mohamed F. Abdelkader and Motaz El Saban, "Car Make and Model recognition combining global and local cues", in proceedings of Twentyfirst International Conference on Pattern Recognition (ICPR), IEEE, 2012.

[12] A. Psyllos, C.N. Anagnostopoulos, E. Kayafas, "Vehicle model recognition from frontal view image measurements", ScienceDirect, 2011.

[13] Gongde Guo, Hui Wang, David Bell, Yaxin Bi, Kieran Greer , "KNN Model-Based Approach in Classification”, Springerlink, 2003.

Engr. Faiza Ayub Syed is a MS scholar as well as Research Associate in the Department of Software Engineering at University of Engineering and Technology Taxila, Pakistan. She graduated from University of Engineering and Technology Taxila in Software Engineering in July 2011. Her areas of interest are Software Design and Architecture, Digital Image Processing, Software Requirement Engineering, Software Qulaity Assurance, Computer Communication and Networks, Web Application Development and Desktop Application Development.

Engr. Malik Usman Dilawar has done his Post Graduation in Computer Engineering from Center for Advance Studies in Engineering, Islamabad, in June 2013. He has recieved his graduation in Electrical Engineering from National Univeristy of Sciences and Technology Rawalpindi in May 2005. His areas of interest are Signal Processing, Image Processing, Computer Communication and Networks, Object Oriented Programming and Web Application Development.

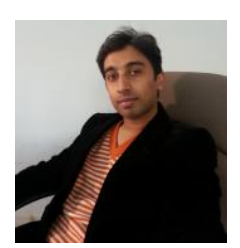

Engr. Ali Javed is serving as an Assistant Professor in the Department of Software Engineering at UET Taxila, Pakistan. He is also a $\mathrm{PhD}$ Scholar in Computer Engineering Department at UET Taxila, Pakistan. He has received his MS degree in Computer Engineering from UET Taxila, Pakistan in February, 2010. He received Chancellor's Gold Medal in MS Computer Engineering degree and became the first MS student in the history of UET Taxila to be awarded Chancellor's Gold Medal. He has received B.Sc. degree in Software Engineering from UET Taxila, Pakistan, in September, 2007. He got 3rd position in Software Batch-2003F in BS Software Engineering degree. His areas of interest are Digital Image Processing, Computer vision, Video Summarization, Mobile Application Development, Software Requirements Engineering, Software Quality Assurance and Software testing.

How to cite this paper: Faiza Ayub Syed, Malik Usman Dilawar, Ali Javed,"Comparative Analysis of Vehicle Make and Model Recognition Techniques", IJIGSP, vol.6, no.4, pp. 60-67, 2014.DOI: 10.5815/ijigsp.2014.04.08 